\title{
Randomly connected cellular automata: A search for critical connectivities
}

\author{
Normand Mousseau \\ Theoretical Physics, University of Oxford \\ 1 Keble Road, Oxford, U.K. OX1 3NP
}

(March 12, 2018)

\begin{abstract}
I study the Chaté-Manneville cellular automata rules on randomly connected lattices. The periodic and quasi-periodic macroscopic behaviours associated with these rules on finite-dimensional lattices persist on an infinitedimensional lattice with finite connectivity and symmetric bonds. The lower critical connectivity for these models is at $C=4$ and the mean-field connectivity, if finite, is not smaller than $C=100$. Autocorrelations are found to decay as a power-law with a connectivity independent exponent $\sim-2.5$. A new intermittent chaotic phase is also discussed.
\end{abstract}

PACS: $05.50,05.45,03.20$ 
There has been some interest, recently, regarding the existence of spatially homogeneous systems displaying periodic or quasi-periodic temporal oscillations [ [] $[5]$. This interest is motivated both by the desire to develop a better understanding of complex dynamical systems and by the need to find simple models representing with some faithfulness dynamical oscillations found in biological and neurophysiological systems [4, [0]. In spite of the fair amount of work already accomplished, the fundamental mechanism at play in simple models like the Chaté-Manneville [1] or the Hemmingsson [2] cellular automata (CA) remains elusive and satisfactory analytical solutions are still missing. I present here a study of the ChatéManneville rules on randomly-connected lattices. These lattices present many advantages. By placing $\mathrm{CA}$ on them, we move from a finite-dimensional problem to an infinite one with finite connectivity. The concept of space, therefore, becomes irrelevant. This removes dimensionality along with the questions of lattice-related effects, choice of neighbours, etc., from the problem, leaving a single parameter: the connectivity. This simplification puts a restriction on the necessary and sufficient conditions to obtain global (quasi-)periodicity. Randomly connected lattices are also closer to many globally periodic biological systems, like neurons or fireflies [4, [5], for example, than the ordered ones.

In this letter, I study the effect of connectivity on the global behaviour of the ChatéManneville type CA. In particular, I search for lower and higher critical connectivities. A lower critical coordination, at which the first cyclic phase appear, is found at $C=4$, a smaller value than what could have been expected from the work on finite-dimensional lattices [7]. As regard the upper critical limit, where a mean-field solution would apply, even with a connectivity as high as 100 quasi-periodic phases can still be found. However, the number of CA rules leading to non-trivial phases peaks at relatively low connectivity, $C=13$, and decreases rapidly as one moves towards higher number of neighbours.

The rules used here were proposed by Chaté and Manneville a few years ago [1]. Since their introduction, these CA have been extensively studied on finite-dimensional lattices 
but they remain imperfectly understood (see, for example, [7 9]). I refer the reader to these papers for more details and will give here only a brief description of the rules. These CA are two-state objects incremented with a parallel dynamics following a totalistic rule given by

$$
s_{i}(t+1)=\left\{\begin{array}{l}
1 \text { if } S_{\min } \leq s_{i}(t)+\sum_{j \in \mathcal{N}_{i}} s_{j}(t) \leq S_{\max } \\
0 \text { otherwise }
\end{array}\right.
$$

where $\mathcal{N}_{i}$ is the neighbourhood of site $i$. In the rest of this paper, I shall use $\mathcal{R}_{S_{\min }-S_{\max }}^{C}$ to denote the rule used, where $C$ is the connectivity of the randomly connected lattice. The macroscopic quantity focused on is mainly the concentration of sites in state $1, c(t)=$ $1 / N \sum_{i} s_{i}(t)$

The construction of a randomly-connected lattice can be pursued following different procedures. If oriented bonds are distributed randomly between the various sites such that if $i$ interacts with $j, j$ does not necessarily interact with $i$ (in the thermodynamical limit, with finite connectivity, the probability of $j$ being also a neighbour of $i$ is zero), the macroscopic quantity $c(t)$ is well described by a second order mean-field solution which takes into account correlations between pairs of nearest-neighbour sites. This result has already been discussed by Chaté and Manneville [7].

Requiring that the bonds be symmetric changes completely this picture. The resulting macroscopic behaviour is strongly non-mean-field like and resemble closely the type of behaviour found on the ordered lattices. In the thermodynamical limit, a randomly connected lattice with a finite connectivity is strictly equivalent to a Cayley tree, i.e. that there exists only a single path between any two points on the network. Because of finite size, this is not true for computer simulations. Keeping open boundaries at the edges is not feasible because on a Cayley tree the number of sites at the boundary is roughly equal to the number of sites in the bulk. Randomly connected lattices are constructed instead by drawing a list of neighbours at random with only two restrictions: a fixed coordination and single bond between any two sites. Doing so, loops of length three and up are implicitly built in the 
network. However, the lack of symmetry (i.e. loops of almost any length are present) should reduce significantly the finite-size effects on the general properties of the CA. To ascertain the validity of this approach, I have performed a series a simulations on networks of different sizes following rule $\mathcal{R}_{2-4}^{4}$, from an average loop length of 5 (256 sites) to 12 (more that 4 million sites). Results show that the temporal behaviour is not affected by the system size except for the usual macroscopic noise which decreases with the number of sites in the model [7]. Although in this work networks with a high connectivity can have an average loop length as low as 4, the preceding results indicate that the behaviour obtained should correspond, at least qualitatively, to what would be found in the thermodynamical limit.

We concentrate first on low and high connectivities. For connectivities from $C=2$ to $C=36$, all the $C(C+1) / 2$ rules were examined starting from a random initial configuration with an equal number of sites in state 0 and 1 . For larger coordination, only the regions in the $S_{\text {min }}-S_{\max }$ plane where non-trivial rules were found at $C=36$ were examined, i.e. around $S_{\min }=S_{\max }=C / 2$. At very low coordination, $C=2$ and 3 , CA do not display any periodic or quasi-periodic cycles. However, for certain rules, the models possess noisy fixed points $(P 1)$. The first cyclic phase appears at $C=4$, where a stable quasiperiodic cycle (rule $\mathcal{R}_{2-4}^{4}$ ) with a period close to 3 (QP3) is found (see Fig. 1). This finding supports claims by Grinstein et al. [3] that on finite-dimensional lattices, it is not the low connectivity generally associated with low dimensions which prevents quasi-periodic cycles to stabilise but really the topology of the space. It shows, also, that finite dimensionality or a symmetric lattice are not necessary to obtain periodicity or quasi-periodicity. We note that the transient period here is extremely short: with a 4 million site network, starting with $c\left(t_{0}=0\right)=0.50$, the CA falls on its cyclic attractor in one time step only. This very short transient is also found for many other rules and connectivities.

The question of the existence of an upper critical dimension for ordered lattices remains unanswered in part because of the lack of a proper theory for these CA and in part because it is numerically difficult, for storage reasons, to go to dimensions higher than 8 or 10 . On a randomly connected lattice, it is much easier to achieve very large connectivity because 
there is no minimum size for the unit cell.

The passage to a mean-field like time evolution appears slowly as one increases the coordination number. Although certain rules still display non-trivial behaviour at $C=22$, others, $\mathcal{R}_{1-2}^{22}$ for example (Fig. (1), follow closely, albeit not exactly, the mean-field solution,

$$
c(t+1)=\sum_{r=S_{\min }}^{S_{\max }} \frac{(C+1) !}{r !(C+1-r) !} c(t)^{r}[1-c(t)]^{C+1-r} .
$$

Because of finite size effects, there exists a threshold for $c(t)$ in the simulation under which the CA will always go to zero. In order to compare the mean-field solution with the simulations, it is useful to introduce a similar cut-off in equation 2. Doing so, it turns out that, for large connectivities, the mean-field rules which do not cross this threshold and, therefore, retain a non-zero $c((t)$ are the only ones leading to non-trivial cycles in the simulation.

However, even reaching connectivities as large as $C=100$, these non-vanishing rules, with $S_{\text {min }}$ just under and $S_{\text {max }}$ just over $C / 2$, still display non-mean field behaviour ( $P 1$ and $Q P 3)$, indicating that if there is a mean-field connectivity, it can only be found at very high coordination. Since it is difficult, for technical reason, to simulate a CA with a connectivity much larger than $C=100$, we have to extract information by following the change in the number and behaviour of non-trivial rules as one varies the coordination number. With increasing coordination, the size of the $Q P 3$ cycle shrinks from a width of about 0.22 with $C=4$ to about 0.03 with $C=100$. Similarly, the center of the cycle moves from $c(t) \sim 0.70$ to 0.49. As shown in Figure 2, the number of non-trivial rules, $n(C)$, for a given connectivity -i.e. rules that are not locally periodic nor in the absorbing state zero - reaches a maximum for $C=13$. It increases almost linearly from $C=2$ and decreases, for large connectivities, roughly as a power-law $n(C) \sim(C-12)^{-0.75}$. For $C=100$, only two rules out of 5050 are found to give non-trivial behaviour. If we use this power-law fit as an indicator, the upper critical connectivity should be in the 100s-range at its lower value. The existence of such a critical value would appear, again from this power-law decrease, to be an artefact of the discrete nature of the rules. If $S_{\min }$ and $S_{\max }$ were continuous, the non-trivial area, in this two-dimensional space, would simply decrease continuously to reach zero only for infinite 
connectivity.

The type of behaviour found in the $S_{\min }-S_{\max }$ diagram for a connectivity between, say, 8 and 14 neighbours is very close to what was found by Chaté and Manneville in 4 to 6 dimensions [0]. The zones identified by these two authors as concentrating $P 1, P 2$ and more complex time behaviour are in extremely good agreement with what is found on these infinite dimensional lattices. However, we see that the $S_{\min }-S_{\max }$ space does not become richer as the connectivity increases beyond $C=13$. From [10], we know that spatial correlation falls as $r_{i j}^{2-d}$, with $r_{i j}$ the distance between two sites. For large $d$, the decay will be so fast that cycles should be dominated by connectivity and not dimensionality. Therefore, the discussion of this paragraph is expected to hold also for finite dimensional lattices: contrary to the predictions of Chaté and Manneville [7], the number of non-trivial rules should become very small in high dimensions.

At low coordination, the randomly-connected CA presents a type of phase which is not found on the ordered lattice: intermittent-chaotic phases displaying a very complex Poincaré map. Rule $\mathcal{R}_{2-6}^{10}$, for example, intermittently goes from a periodic two to a wide-band chaotic behaviour (Fig. 3(a)). Moreover, as can be seen from Fig. 3(b), this cycle not belong to mean-field solutions.

As regard the stability of the phases discussed here, introduction of external noise and internal frustration show that they are as stable as the CA on an ordered lattice. External noise was introduced following the protocol described in [11 and cycles persist with finite amount of random spin inversion after each timestep. The effect of frustration will be discussed elsewhere but cycles remain stable under finite amount of it.

Finally, a word about the decay in the site auto-correlation function,

$$
S\left(t, t_{0}\right)=\frac{1}{N} \sum_{i} s_{i}(t) s_{i}\left(t_{0}\right)-c(t) c(t+1)
$$

It has been recently proposed that for finite-dimensional CA, rules producing quasi-periodic cycles show a power-law decay going as $\left(t-t_{0}\right)^{-(d-2) / 2}$. For the randomly-connected lattice, autocorrelations also decay as a power law. However, the exponent, $\simeq-2.5$, seems to be 
independent of the connectivity as well as the type of non-trivial cyclic behaviour (Fig. 5). The meaning of this exponent still remains to be explained.

In this letter, I have established a lower critical connectivity for the Chaté-Mannevilletype CA on a randomly-connected lattice. Results seem to indicate also the presence of a higher critical connectivity, due to the discreteness of the problem, which should be in the region of a few hundred neighbours. From work on finite-dimensional lattices, it was conjectured that the complexity of the cycles would increase with the dimensionality of the space to break down only when reaching $d=\infty$ [7]. This ever-increasing complexity does not seem to happen. There is a peak in the number of non-trivial phases at a finite connectivity $C=13$ followed by a rapid decrease in this number as connectivity is increased. Because for high dimensional lattices the spatial correlation decay is expected to be fast, results obtained here should be qualitatively applicable to those in this limit.

These results improve on the general understanding about what are the fundamental ingredients needed to obtain non-trivial periodic or quasi-periodic behaviour. In particular, (1) symmetric bonds are necessary to obtain non-mean field behaviour. However, no other loops are needed. (2) Particular topology or symmetries for the network are not needed. (3) The lower connectivity is 4 while the higher critical one should be very large (at least, a few hundred neighbours), if is exists at all. These results could also be applied to biological problems where the effective dimensionality is high.

\section{ACKNOWLEDGEMENTS}

I would like to acknowledge helpful discussions with G.T. Barkema and D. Sherrington. I would also like to thank the Natural Sciences and Engineering Research Council of Canada for a post-doctoral fellowship. 


\section{REFERENCES}

[1] Chaté H. and Manneville P., Europhys. Lett., 14 (1991) 409.

[2] Hemmingsson J., Physica A, 183 (1992) 255.

[3] Grinstein G., Mukamel D., Seidin R. and Bennett Ch., Phys. Rev. Lett. 70 (1993) 3607.

[4] Herz A. V. M. and Hopfield J. J., Phys. Rev. Lett. 75 (1995) 1222.

[5] Bottani S., Phys. Rev. Lett. 74 (1995) 4189.

[6] Buck J., Quart. rev. Bio. 63 (1988) 265.

[7] Chaté H. and Manneville P., Prog. Theo. Phys. 87, (1992) 1.

[8] Gallas J. A. C., Grassberger P., Herrmann H. J. and Ueberholz P., Physica A, 180 (1992) 19.

[9] Mousseau N., Europhys. Lett. 28, 551 (1994) and Ibid. 29, 269 (1995) (Erratum).

[10] Chaté H., G. Grinstein and L.-H. Tang, Phys. Rev. Lett. 74, 912 (1995).

[11] Mousseau N., to be published. 


\section{FIGURES}

FIG. 1. Magnetisation map for an automata following $\mathcal{R}_{2-4}^{4}$. One thousand timesteps on a system with 4194304 sites. The transient here is one timestep.

FIG. 2. Number of rules leading to non-trivial time behaviour as a function of connectiviy. A non-trivial rules is one which does not lead the system to zero or local periodic states. The lines are guide to the eyes and are described in the text.

FIG. 3. (a) Time evolution of the macroscopic $c(t)$ for intermittent chaotic-periodic rule $\mathcal{R}_{2-6}^{10}$; 500000 sites, 20000 timesteps. (b) Poincaré map for the same rule. The line is the mean-field solution.

FIG. 4. For large connectivity, the magnetisation map is close to the mean-field solution. Here, $\mathcal{R}_{2-12}^{22}$ on a 500 000-sites lattice. The line is the mean-field solution.

FIG. 5. Auto-correlation as a function of time for $\mathcal{R}_{2-4}^{4}$ (short dashes), $\mathcal{R}_{2-4}^{10}$ (solid line) and

$\mathcal{R}_{9-12}^{22}$ (long dashes), respectively a $Q P 3, P 2 \times Q P 3$ and $Q P 3$ cycles. The straight line is a guide to the eye, with a slope of -2.5 . 


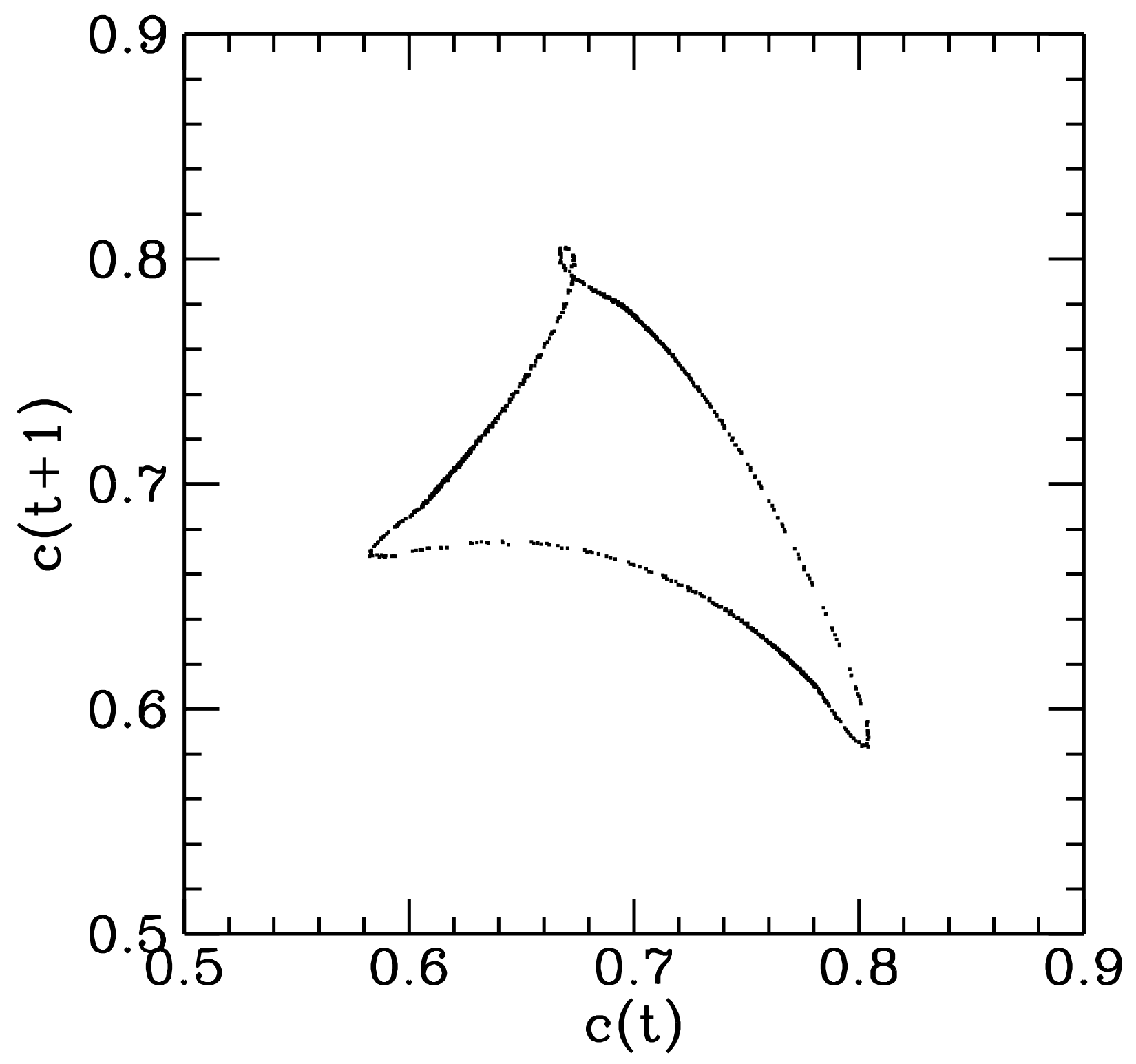




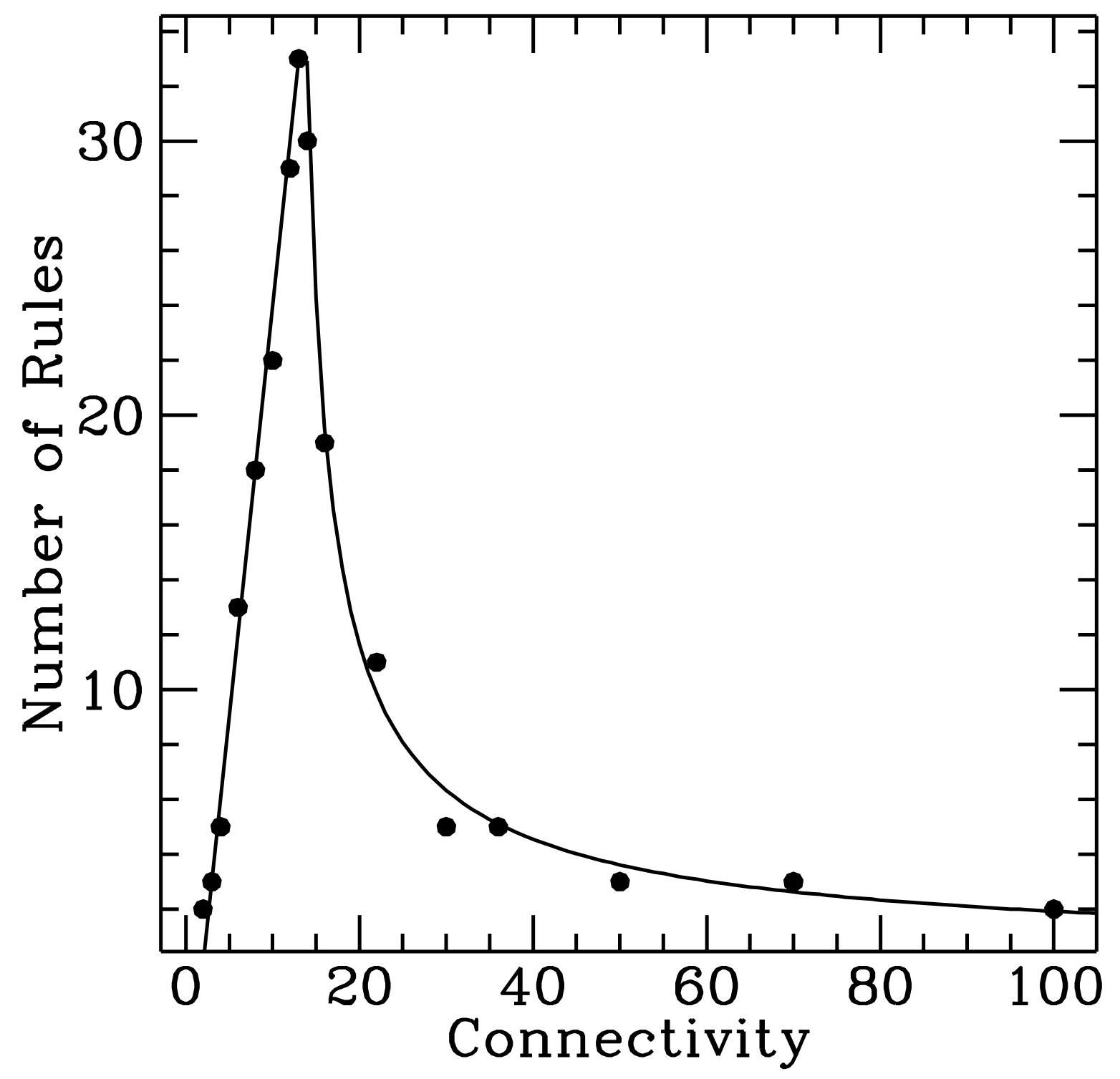




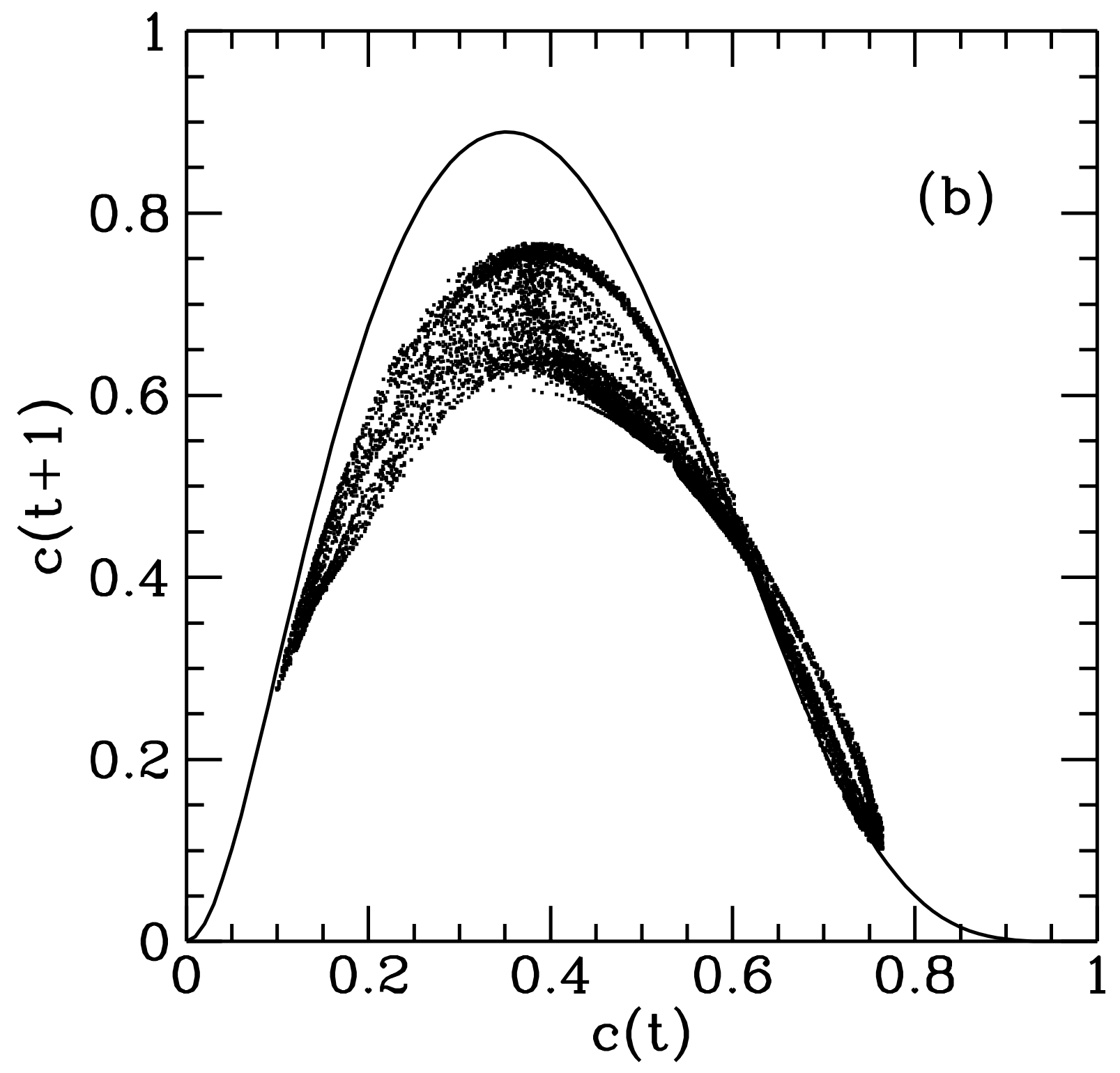




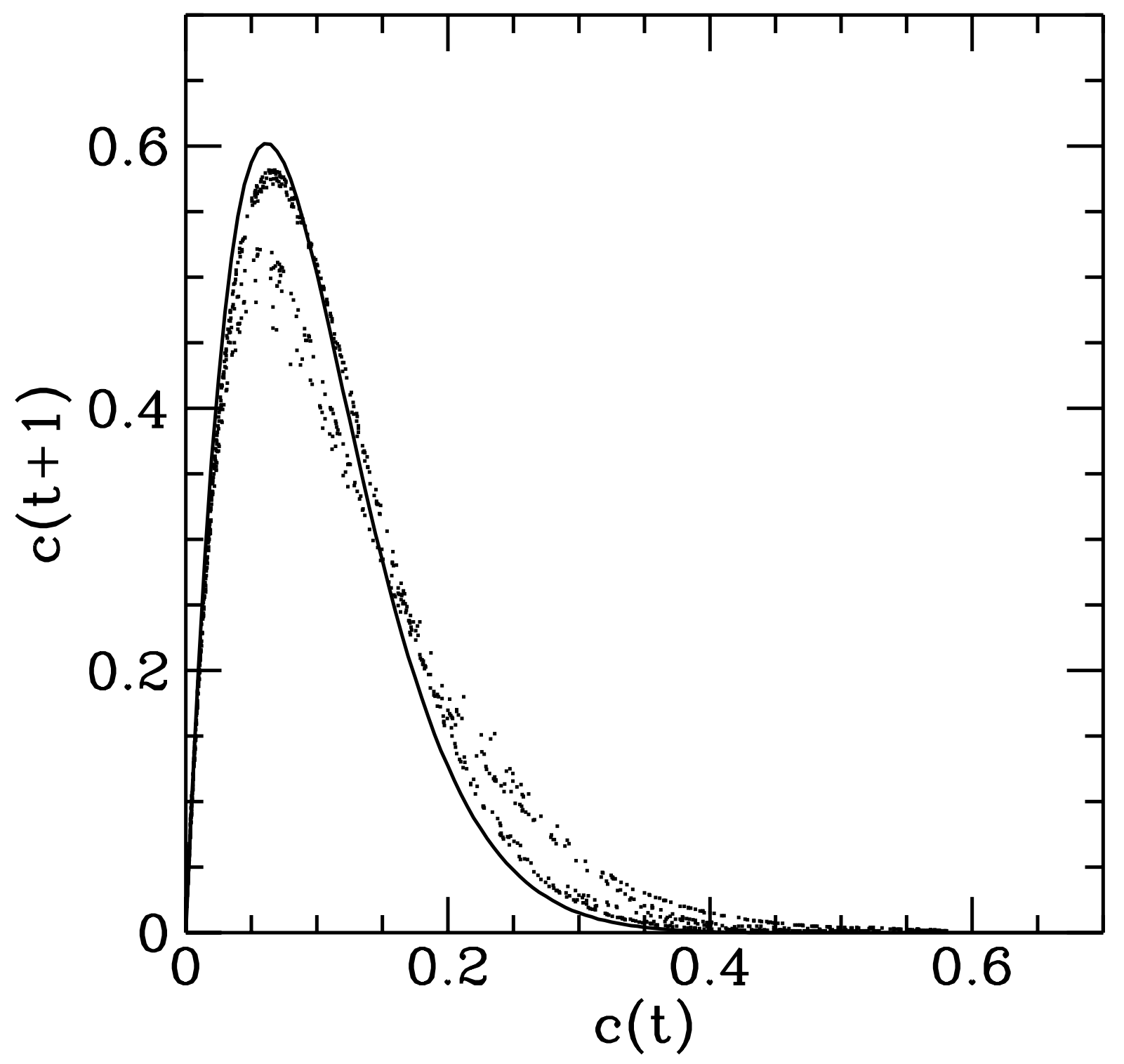




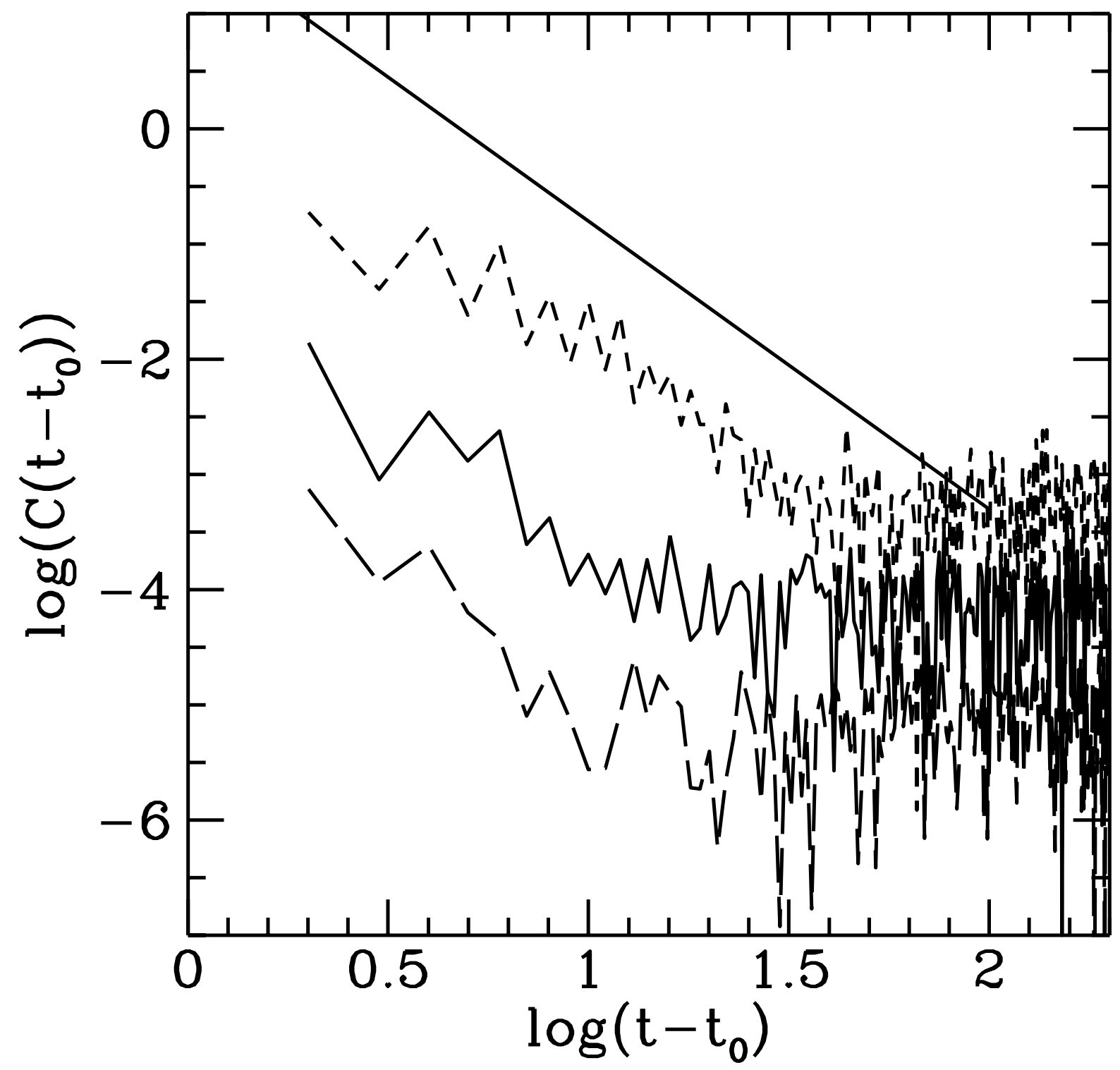

